\title{
Schwangerschafts-Granulom bei einer 37-jährigen 1. Gravida, 1. Para - ein Fallbericht
}

\section{Granuloma Gravidarum in a $37-y e a r-o l d ~ 1^{\text {st }}$ Gravida, $1^{\text {st }}$ Para - A Case Report}

\author{
Autor \\ S. Findeklee \\ Institut \\ Gynecology and Obstetrics, Universitätsklinikum Magdeburg, Magdeburg
}

Schlüsselwörter

- Schwangerschafts-Granulom

- Prophylaxe

- Therapie

- Prävention

Key words

- granuloma gravidarum

- prophylaxis

- therapy

- prevention eingereicht $\quad 02.11 .2014$ angenommen 29.01.2015 nach Überarbeitung

\section{Bibliografie}

DOI http://dx.doi.org/ $10.1055 / \mathrm{s}-0035-1545342$

Online-Publikation: 24.9.2015

Z Geburtsh Neonatol 2015;

219: $235-237$

(c) Georg Thieme Verlag KG Stuttgart · New York ISSN 0948-2393

\section{Korrespondenzadresse \\ Dr. Sebastian Findeklee}

Gynecology and Obstetrics Universitätsklinikum Magdeburg Gerhart-Hauptmann-Straße 35 39108 Magdeburg Tel.: + 49/391/6717 399 Fax: +49/391/6717 311 sebastian.findeklee@arcor.de

\section{Zusammenfassung}

$\nabla$

Das Schwangerschafts-Granulom ist ein seltener, vom Zahnfleisch (Gingiva) ausgehender gutartiger Tumor. Es kommt bei 0,2-14\% aller Schwangerschaften vor. Meist verläuft die Erkrankung asymptomatisch und wird infolge der hormonellen Veränderungen nach der Geburt terminiert. Sollte das Granulom Beschwerden bei der Schwangeren wie z.B. starke Schmerzen beim Kauen oder rezidivierende Blutungen, so kommt therapeutisch neben operativen und nichtoperativen Maßnahmen die Entbindung infrage. Wir schildern den Fall einer 37-jährigen 1. Gravida, 1. Para, die in der 39/2 Schwangerschaftswoche (SSW) wegen eines symptomatischen Schwangerschafts-Granuloms vorzeitig entbunden werden musste. Postpartal kam es innerhalb von 3 Monaten ohne Intervention zur Remission des Schwangerschafts-Tumors. Der Fallbericht verdeutlicht die Wichtigkeit der Aufklärung Schwangerer über die Bedeutung einer oralen Hygiene sowie regelmäßiger zahnärztlicher Kontrolluntersuchungen zur Prophylaxe eines Schwangerschafts-Granuloms.

\section{Einleitung \\ $\nabla$}

Das Schwangerschafts-Granulom, auch Granuloma oder Epulis gravidarum genannt, ist ein seltener, bei ca. einer von 500 Schwangeren auftretender benigner Tumor. Seine Pathogenese gilt bis heute als nicht vollständig verstanden. Zentral an seiner Entstehung beteiligt sind Schwangerschafts-induzierte Gefäßveränderungen sowie eine vermehrte Expression bzw. Wirksamkeit von Wachstumsfaktoren wie VEGF (vascular endothelial growth factor) oder FGF (fibroblast growth factor) [1]. Im Allgemeinen gelten diese Veränderungen nach Beendigung der Schwangerschaft als reversibel. Nichtsdestotrotz gibt es

\section{Abstract}

$\nabla$

The granuloma gravidarum is a rare benign tumour with gingival origin. It occurs in circa $0.2 \%$ of pregnancies. Mostly we see an asymptomatic course of disease terminated by hormonal changes after delivery. If the granuloma is associated with complaints of the pregnant woman, for example masticational pain or recurrent bleedings, therapeutic options are conservative therapy, surgery or delivery. We report the case of a $37-$ year-old $1^{\text {st }}$ gravida, $1^{\text {st }}$ para who had an induced delivery in the $39+2$ gestational week because of a symptomatic granuloma gravidarum. We saw a spontaneous remission of the granuloma within 3 months post partum. The case report underlines the importance of suitable information for pregnant women about oral hygiene and the necessity of regular dental controls during pregnancy for prophylaxis of granuloma gravidarum.

auch Fallberichte über ein-auch nach der Entbindung-rezidivierendes Granulom [2]. Zu den Hauptrisikofaktoren für die Entstehung eines Schwangerschafts-Granuloms zählt eine unzureichende Mundhygiene [3]. Eine zahnärztliche Kontrolluntersuchung gehört damit auch obligat zur Schwangerschaftsvorsorge.

\section{Fallbericht \\ $\nabla$}

Wir berichten über eine 37-jährige 1. Gravida, 1. Para, die sich erstmals am 16.04.2014 in der rechnerisch 39/1 SSW in der Intensivschwangerenberatung unserer Klinik vorstellte. Die Über- 


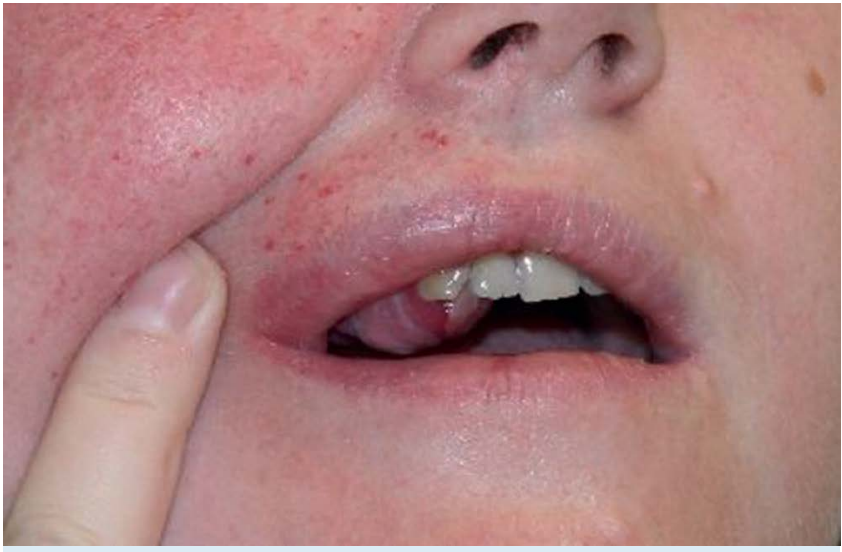

Abb. 1 Äußerer Befund bei der stationären Aufnahme.

weisung erfolgte durch die behandelnde Frauenärztin wegen eines seit 2 Tagen progredienten Exanthems im Gesichtsbereich. Anamnestisch berichtete die Patientin über seit 14 Tagen bestehendes Zahnfleischbluten sowie eine zunehmende Schwellung der rechten Wange $\boldsymbol{\bullet}$ Abb. $\mathbf{1}$ und 2. An Vorerkrankungen fanden sich eine leichte Myopie ( $-1,25$ Dioptrien beidseits) und multiple Nahrungsmittelallergien (Soja, Sojalecithin, Haselnuss, Mandel, Apfel, Birne, Aprikose, Brombeere, Himbeere, Litschi, Kirsche, Kiwi, Pfirsich, Pflaume, Tomate, Hafer). Bei der Untersuchung des Lokalbefunds zeigte sich eine exophytische Raumforderung der Mundschleimhaut des rechten Oberkiefers (siehe Foto). Der geburtshilfliche Befund war unauffällig. Sonografisch zeigten sich ein zeitgerecht entwickelter Fet in Schädellage, normale Fruchtwasserverhältnisse und eine Plazenta-Reife ${ }^{\circ}$ I. Die Portio erschien weich, $1 \mathrm{~cm}$ erhalten und für einen Finger einlegbar.

Es erfolgte eine konsiliarische Vorstellung in unserer MundKiefer-Gesichtschirurgie. Hier wurde die Diagnose Schwangerschafts-Granulom gestellt und therapeutisch die Entbindung bzw. ggf. postpartal eine elektrochirurgische Abtragung der exophytischen Schleimhaut empfohlen. Wir begannen zudem am gleichen Tag mit einer antibiotischen Behandlung mit Amoxicillin $3 \times 1 \mathrm{~g}$ oral für 7 Tage und empfahlen eine Intensivierung der Mundpflege. Nach der konsiliarischen Vorstellung entschieden wir uns aufgrund des erheblichen Leidensdrucks der Patientin infolge stark eingeschränkter Fähigkeit zur Nahrungsaufnahme und bei noch unreifem Muttermundsbefund (Bishop-Score $\mathbf{0}$ Punkte) für eine Entbindung per Sectio in Spinalanästhesie am 17.04.2014 in der 39/2 SSW. Es wurde ein lebensfrischer Junge mit einem Gewicht von $3825 \mathrm{~g}$ und einer Körperlänge von $53 \mathrm{~cm}$ geboren. Der APGAR betrug 9/10/10 und der NabelarterienpH-Wert 7,24.

Die Patientin wurde bis zum 5. postpartalen Tag (22.04.2014) an unserer Klinik betreut. Bei den täglichen Visiten zeigte sich bereits am 1. Tag nach der Entbindung ein diskreter Rückgang des Gesichts-Exanthems und der Schwellung der rechten Wange und die Wöchnerin berichtete über eine leichte Besserung ihrer krankheitsbedingten funktionellen Einschränkungen. Am 22.04.2014 veranlassten wir eine erneute Vorstellung in der Mund-Kiefer-Gesichts-Chirurgie. In diesem Zusammenhang wurde ein Angio-CT der rechten Wange, des Kopfs und des Halses durchgeführt. Dieses ergab folgende Befunde: Es zeigte sich ein durch Flüssigkeit subtotal verlegter rechter Sinus maxillaris, der ansonsten unauffällig erschien. Es fand sich ein geringgradig vermehrter Lymphknotenbesatz entlang der Gefäßnervenstraßen rechts. Die übrigen anatomischen Strukturen stell-

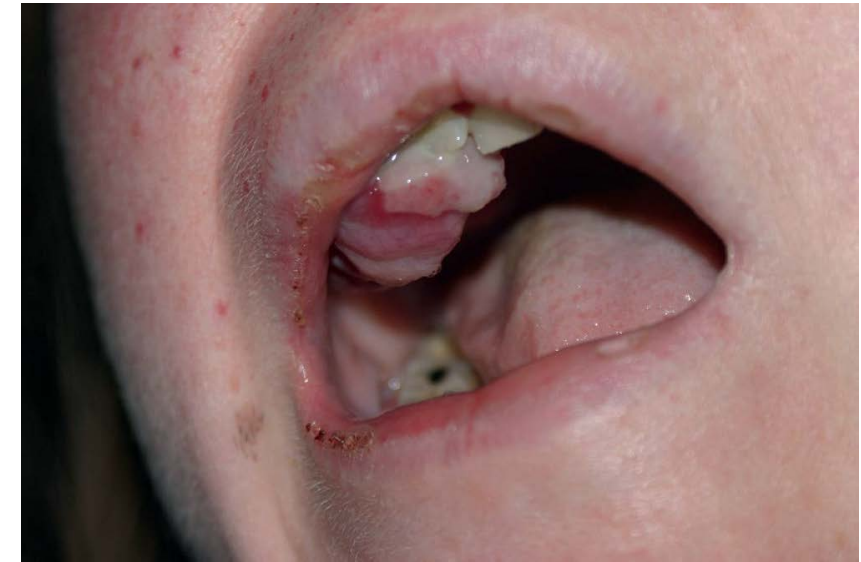

Abb. 2 Oraler Befund zum Aufnahmezeitpunkt.

ten sich unauffällig dar. Insbesondere ergab sich kein Hinweis auf eine umschriebene Raumforderung.

Am 22.04.2014 (5. postpartaler Tag) konnte die Patientin mit unauffälligem geburtshilflichem Befund aus unserer Klinik entlassen werden. Eine ambulante Wiedervorstellung in der Klinik für Mund-Kiefer-Gesichts-Chirurgie mit den Befunden der Angio-CT-Untersuchung wurde für den 25.04.2014 vereinbart. Hier zeigte sich der Lokalbefund im Vergleich zum Vorbefund leicht regredient. Es wurden 14-tägige Kontrolltermine in der Ambulanz der Klinik für Mund-, Kiefer- und Gesichtschirurgie vereinbart, die unsere Patientin auch wahrnahm. Innerhalb von 3 Monaten kam es ohne spezifische Therapie zum Abheilen des Schwangerschafts-Granuloms.

\section{Diskussion}

$\nabla$

Das Schwangerschafts-Granulom stellt eine spezielle, lokalisierte Form der Gingivitis dar. Histologisch besteht kein Unterschied zum außerhalb der Schwangerschaft vorkommenden pyogenen Granulom, weshalb beide Begriffe synonym verwendet werden. Es handelt sich beim Schwangerschafts-Granulom um eine gutartige Läsion des Zahnfleischs (insbesondere der interdentalen Papillen) mit Vorkommen an Mundschleimhaut und Lippen. Klinisch imponiert es als kleiner, weicher, rötlicher, glatter oder gelappter, exophytischer Tumor auf einer gestielten oder festen Basis, der sich innerhalb weniger Tage oder Wochen entwickelt. Das Schwangerschafts-Granulom besitzt eine gute Vaskularisation und blutet daher leicht. Im Ruhezustand ist es meist schmerzlos. Beim Kauen können jedoch Schmerzen auftreten $[4,5]$.

Die Pathogenese des Schwangerschafts-Granuloms ist noch immer nicht abschließend geklärt. Neben Defiziten bei der Mundhygiene werden auch hormonelle Faktoren als ursächlich genannt. So postulierten Mealey et al., dass erhöhte Östrogenund Progesteronspiegel während der Schwangerschaft die Konzentration des pathogenetisch bedeutsamen Bakteriums Prevotella intermedia im subgingivalen Biofilm erhöhen. Des Weiteren könnten erhöhte Konzentrationen von Östrogen und Progesteron für eine Minderung der Resistenz im Bereich des bakteriellen Biofilms, eine Erhöhung der Gefäßpermeabilität, ein Eindringen von Flüssigkeiten in die perivaskulären Gewebe und eine verstärkte Entzündungsreaktion verantwortlich, wodurch die Entwicklung einer granulomatösen Läsion begünstigt wird $[6,7]$. Unabhängig davon waren in einer von Cardoso et al. veröffentlichten retrospektiven Studie, in der zwischen 1980 
und 201241 Frauen mit Schwangerschafts-Granulom in Brasilien untersucht wurden, der bakterielle Biofilm und von den Zähnen ausgehende Konkremente als Grundlage für eine Entzündungsreaktion im Bereich des Zahnfleischs durch ihre lokal irritative Wirkung die wichtigsten die Entstehung von Schwangerschafts-Granulomen begünstigenden Faktoren [8]. Insofern verwundert es nicht, dass sich in der Literatur vielfach die Empfehlung findet, Schwangere über die Notwendigkeit regelmäßiger zahnärztlicher Kontrolluntersuchungen und einer suffizienten Mundhygiene aufzuklären. Laut Krishnan et al. würden sich alleine durch einfache orale Hygieneregeln (v.a. Entfernung von Plaque, Benutzung einer weichen Zahnbürste) die meisten schwangerschaftsbedingten Zahnfleisch-Läsionen problemlos vermeiden lassen. Unabhängig von der Notwendigkeit einer generellen Mundhygiene mit der Meidung lokal reizender Substanzen wie Nikotin während der gesamten Schwangerschaft wird darauf hingewiesen, dass im Falle eines manifesten und symptomatischen Granuloms der günstigste Zeitpunkt für eine chirurgische oder zahnärztliche Behandlung das 2. Trimenon ist. Zugleich betonen Krishnan et al., dass Zahnfleisch-Veränderungen während der Schwangerschaft selten einer chirurgischen Therapie bedürfen und postpartal bis auf Einzelfälle eine spontane Rückbildungstendenz zeigen [9]. Bei der Therapie des Schwangerschafts-Granuloms lässt sich ein nicht-operatives von einem operativen Vorgehen unterscheiden. Im Rahmen einer operativen Therapie kommt die sogenannte periodontal plastic surgery zum Einsatz. Diese beinhaltet die Entfernung des gingivalen Tumors durch Abfräsen sowie eine Abdeckung des Defekts mit Mukosa. Die Evidenz für dieses Verfahren ist jedoch gering und gründet sich auf Fallberichte [10]. Weitere Fallstudien existieren zur Behandlung des Schwangerschafts-Granuloms mittels NdYAG-Laser und Infrarot-Laser [11,12]. Gleichzeitig wiesen Sills et al. darauf hin, dass eine Behandlung des Schwangerschafts-Granuloms, das eine gute postpartale Rückbildungstendenz aufweist, meist nicht nötig ist, insbesondere wenn das Granulom klein sowie schmerz- und blutungsfrei imponiert [13]. Auch bei der von uns behandelten Patientin kam es nach der Entbindung zu einer deutlichen Remission des Schwangerschafts-Granuloms. Während der Schwangerschaft erfolgte keine zahnärztliche Konsultation. Ein Nikotinabusus oder die Einnahme von das Zahnfleisch angreifenden Substanzen ließen sich nicht eruieren.

Genaue Kriterien für die Indikation zur Entbindung von Frauen mit Schwangerschafts-Granulom lassen sich in der Literatur nicht finden. Bei unserer Patientin gab die starke subjektive Beeinträchtigung mit fast vollständiger Unfähigkeit zur oralen Nahrungsaufnahme den Ausschlag zur Entbindung. Gleichwohl sollte eine vorzeitige Entbindung nur nach sorgfältiger Abwägung von Nutzen und Risiken erfolgen.

\section{Fazit}

$\nabla$

Das Schwangerschafts-Granulom ist ein insgesamt zwar seltenes Krankheitsbild, das jedoch in ausgeprägten Stadien zu erheblichen Beschwerden führen und eine Schwangerschaftsbeendigung erforderlich machen kann. Hätte bei der von uns behandelten Patientin eine frühere Evaluation des oralen Status mit verbesserten oralen Hygienemaßnahmen stattgefunden, so wären das Wachstum des Granuloms und die damit einhergehenden Beschwerden sowie die Sectio wahrscheinlich vermeidbar gewesen. Unser Fallbericht zeigt, dass bei der Schwangerenbetreuung immer die Frau als „Ganzes“ gesehen werden muss und neben der notwendigen Untersuchung des geburtshilflichen Befunds die Zahngesundheit nicht außer Acht gelassen werden darf.

\section{Interessenkonflikt}

$\nabla$

Es besteht kein Interessenkonflikt.

\section{Literatur}

1 Bragado $R$, Bello E, Requena $L$ et al. Increased expression of vascular endothelial growth factor in pyogenic granulomas. Acta Derm Venereol 1999; 79: 422-425

2 Reddy NR, Kumar PM, Selvi T et al. Management of Recurrent Post-Partum Pregnancy Tumor with Localized Chronic Periodontitis. Int J Prev Med 2014; 5: 643-647

3 Terezhalmy GT, Riley CK, Moore WS. Pyogenic granuloma (pregnancy tumor). Quintessence Int 2000; 31: 440-441

4 Rath W, Gembruch U, Schmidt S, Hrsg. Geburtshilfe und Perinatalmedizin. 2. Auflage. Thieme-Verlag, Stuttgart: 2010; 150

5 www.uptodate.com/pyogenic-granuloma

6 Mealey BL, Moritz AJ. Hormonal influences: effects of diabetes mellitus and endogenous female sex steroid hormones on the periodontium. Periodontol 2000 2003; 32: 59-81

7 Mascarenhas P, Gapski R, Al-Shammari K et al. Influence of sex hormones on the periodontium. J Clin Periodontol 2003; 30: 671-681

8 Cardoso JA, Spanemberg JC, Cherubini K et al. Oral granuloma gravidarum: a retrospective study of 41 cases in Southern Brazil. J Appl Oral Sci 2013; 21: 215-218

9 Krishnan B, Arunprasad G, Madhan B et al. Giant granuloma gravidarium of the oral cavity. BMJ Case Rep 2014, doi:10.1136/bcr-2014204057

10 Cruz LE, Martos J. Granuloma gravidarum (pyogenic granuloma) treated with periodontal plastic surgery. Int J Gynaecol Obstet 2010; 109: 73-74

11 Powell JL, Bailey CL, Coopland AT et al. NdYAG laser excision of a giant gingival granuloma of pregnancy. Lasers Surg Med 1994; 14: 178-183

12 Meffert JJ, Cagna DR, Meffert RM. Treatment of oral granulation tissue with the flashlamp pulsed dye laser. Dermatol Surg 1998; 24: 845-848

13 Sills ES, Zegarelli DJ, Hoschander MM et al. Clinical diagnosis and management of hormonally responsive oral pregnancy tumor (pyogenic granuloma). J Reprod Med 1996; 41: 467-470 